\title{
Tangence
}

\section{La langue de Ringuet ne parle pas : elle écrit}

\section{Réjean Beaudoin}

Numéro 40, mai 1993

Régionalismes littéraires de la francophonie

URI : https://id.erudit.org/iderudit/025765ar

DOI : https://doi.org/10.7202/025765ar

Aller au sommaire du numéro

Éditeur(s)

Tangence

ISSN

0226-9554 (imprimé)

1710-0305 (numérique)

Découvrir la revue

Citer cet article

Beaudoin, R. (1993). La langue de Ringuet ne parle pas : elle écrit. Tangence, (40), 39-48. https://doi.org/10.7202/025765ar d'utilisation que vous pouvez consulter en ligne.

https://apropos.erudit.org/fr/usagers/politique-dutilisation/ 


\section{La langue de Ringuet ne parle pas: elle écrit}

\section{Réjean Beaudoin *}

Commençons par quelques paradoxes. Ils valent, me semblet-il, la réalité des clichés qui les composent.

Le premier roman de Ringuet, Trente arpents, paru en 1938, fait entendre le chant du cygne de l'école du terroir. Le roman québécois s'ouvre à l'expression de la réalité urbaine ${ }^{1}$ par un roman de la terre. Un événement de cette importance se produit dans un livre publié chez Flammarion, à Paris, ce qui est peutêtre dans l'ordre des choses puisque Maria Cbapdelaine était sorti d'une plume française. Quant à l'anti-régionalisme déclaré de l'auteur ${ }^{2}$ de Trente arpents, il remonte à sa jeunesse de carabin, lorsque l'étudiant en médecine fréquentait Victor Barbeau et Marcel Dugas parmi les Casoars de l'Arche et qu'il publiait des poèmes exotiques sous le pseudonyme du Sphinx d'Halifax! Les amusants pastiches écrits avec Louis Francœur dans Littératures... à la manière de... ${ }^{3}$ laissent assez voir de quel côté se situait Philippe Penneton dans le débat parfois virulent suscité par les défenseurs de «l'heure des vaches", pour reprendre la robuste

- Réjean Beaudoin est professeur à l'Université de Colombie-Britannique. Il a publié Naissance d'une littêrature. Essai sur le messianisme et les débuts de la littérature canadienne-française (1989) et Le roman québécois (1991).

1 L'œuvre du romancier s'accorde avec cette rupture: Fausse monnaie, en 1947, et surtout Le poids du jour, en 1949, sont des romans urbains. Roger Lemelin et Gabrielle Roy avaient marqué ce virage dans l'évolution du roman québécois vers la fin de la Seconde Guerre mondiale.

2 "Ce livre n'est pas un roman "régionaliste"; les paysans que j'ai connus n'étaient pas des héros. Ce livre n'est pas un roman "naturaliste"; les paysans que j'ai connus n'étaient pas des brutes. * Cette phrase vient de la page de titre du manuscrit de Trente arpents cité par Jean Panneton dans son Ringuet, Montréal, Fides, coll. *Ecrivains canadiens d'aujourd'hui *, 1970, p. 43. Jean Panneton ajoute: *En réaction contre la tendance régionaliste qui menait toujours à la pastorale, Trente arpents voulut présenter notre habitant comme un être peu poétique, penché sur quelques arpents de terre ingrate, dont l'existence semblait soumise à une dure fatalité. Il est évident que Ringuet n'a pas voulu peindre les charmes de la vie primitive. (p. 47).

3 Montréal, Édouard Garand, 1924. 
40

expression de l'auteur de "La danse autour de l'érable "4́ L'ironie du sort, c'est que Trente arpents ait pu être lu comme la forme accomplie du roman régionaliste. La réception critique canadiennefrançaise de l'époque en témoigne sans ambiguïté chez un Claude-Henri Grignon. Le vibrant hommage qu'il rend à Ringuet annonce ses couleurs dans le titre de l'article: "Les trente arpents d'un canayen ou le triomphe du régionalisme " 5 . Louis Dantin souligne, pour sa part, combien l'œuvre est "intensément française ", mais il n'en insiste pas moins sur le miroir qu'elle offre à "nos traits distinctifs, nos types originaux, nos mœurs intimes, notre âme secrète * et "notre mentalité laurentienne ${ }^{6}$. Comme l'écrit l'auteur des Confidences, *le paradoxe, ennuyeux quand il est grave, devient savoureux quand il est offert avec le sourire" 7 . L'ennui est justement que ces critiques n'entendent pas du tout badiner. Tout se passe comme s'il s'agissait de colmater immédiatement la brèche aperçue entre réalisme et régionalisme.

Le traitement des dialogues sous la plume du romancier n'est pas davantage exempt de contradictions. Il faudra y revenir, mais il n'est pas inutile de rappeler d'abord comment Philippe Panneton appréciait la diction rocailleuse des habitants qu'il fait parler dans son premier roman. Des textes inédits (mais bien connus, parce que souvent cités) attestent que l'écrivain avait en horreur la façon de s'exprimer de ses compatriotes:

Mais à ceux qui me diront que les Canadiens ne parlent pas si mal, je saurai dorénavant répondre. Souventes fois, je n'ai pu me faire comprendre qu'en traduisant du français en patois, c'est-à-dire en prenant l'accent, les formes verbales et les constructions des clients, surtout paysans. ${ }^{8}$

4 Texte polémique de Victor Barbeau, farouche adversaire de l'indigénisme attribué à l'école régionaliste.

5 Valdombre (pseudonyme de Claude-Henri Grignon) n'hésite pas à écrire, à propos de Trente arpents, que “c'est la découverte du roman régionaliste canadien-français, non pas un régionalisme mièvre à la Blanche Lamontagne, mais plutôt un essai brutal vers l'objectivité, la ligne drue, la ligne paysanne, la ligne droite ", Les pamphlets de Valdombre, $3^{-}$ année, $\mathrm{n}^{\circ} 3$, février 1939 , p. 122.

6 Louis Dantin, L'avenir du Nord, 3 mars 1939, cité dans Jean Panneton, Ringuet, Montréal, Fides, 1970, p. 173.

7 Ringuet, Confidences, Montréal, Fides, 1965, p. 101.

8 Journal (inédit) de Philippe Panneton, cité par Jean Panneton dans Ringuet, op. cit., p. 48. 
Le mot qui frappe ici, c'est le mot traduire. Le docteur Panneton se dit forcé, dans sa pratique médicale, de traduire ( "du français en patois") ce qu'il veut faire comprendre à sa clientèle paysanne. Les malades parlent d'une façon qui détonne à l'oreille du clinicien - cela va de soi -, mais cette divergence est telle qu'elle est rapportée à l'incompatibilité de deux langues différentes. L'importance de la coupure est extraordinaire. Mais quel est donc la langue parlée par le docteur Panneton? "Souventes fois...", écrit-il. L'élégante tournure archaïque signale la teneur littéraire de son discours. L'expression est admirablement choisie pour noter pareil écart. On comprend que la communication doive passer par un changement de code si le disciple d'Esculape s'exprime de la sorte avec les Euchariste qui viennent le consulter pour un tour de rein. S'agit-il pour autant d'une autre langue? La distance du médecin lettré au patient analphabète n'est certainement pas celle qui sépare le français d'une langue étrangère, mais la distribution normale de l'oral et de l'écrit dans la même langue. La nuance est de taille. On verra que cette posture paradoxale est intimement liée chez Ringuet à la pratique de l'écriture. On aura remarqué d'ailleurs la beauté de son raisonnement: l'excommunication de la parole des paysans s'autorise du rejet de leur langue réputée étrangère au français; cette nouvelle langue, le paysan canayen, le docteur Panneton la connaît suffisamment pour la parler au besoin avec ses patients (on se demande où il a pu perfectionner sa maîtrise de cet idiome), mais ill n'y parvient qu'au prix de se traduire lui-même en dialecte! Comme si, du bourgeois trifluvien au fermier de la Pointe-du-Lac, où la famille Panneton passait la saison estivale, on franchissait une frontière linguistique inscrite dans la structure sociale. Cette façon de voir est singulière.

On m'objectera que je force la portée du témoignage en feignant d'ignorer la possibilité d'un usage métaphorique du mot "traduire "dans ce texte: l'auteur aurait seulement voulu dire, en somme, qu'il lui fallait changer de niveau de langue pour être compris de ses interlocuteurs ruraux. Pourtant, les deux phrases ne laissent subsister aucun doute sur le contexte de la communication verbale qui oblige à donner son plein sens à la traduction dont il est question. Le statut professionnel et la cornpétence linguistique sont curieusement articulés dans l'observation qui prétend établir la surdité du. parler populaire à l'intelligence de la langue française. Cette position du docteur relègue l'élocution 
indigène au rang de perturbation exogène. Le jugement emprunte la rigueur du diagnostic et le traitement ressemble à celui que les défenseurs de la langue française appliquaient traditionnellement à l'anglicisme assimilé à une maladie. Il faut prendre ce texte au pied de la lettre. Le refus du régionalisme et l'appartenance de classe se touchent dans ce commentaire. L'écrivain et le médecin y sont solidaires dans l'affirmation qui les élève au-dessus de la parlure rustique. Puisqu'il s'agit d'aligner les paradoxes, la réversibilité de cette étrange attitude rendrait peut-être son sens plus accessible: se pourrait-il que sa formation européenne et sa fonction sociale aient rendu l'écrivain sourd au patois campagnard? Si tel était le cas, "l'accent, les formes verbales et les constructions des clients, surtout paysans " seraient alors passibles d'une traduction littéraire, seul moyen de les faire entendre à celui qui les considère en fait comme les sonorités discordantes d'une langue inconnue. Il faut bien remarquer, dans cette hypothèse, le renversement de la déclarationn littérale: l'habitant malade peut fort bien comprendre le français du docteur, mais celui-ci refuse par contre de prêter toute signification aux mots (qui ne sont que des maux) des patients. Ni son savoir médical ni son art d'écrire ne sauraient en tenir compte.

Je tiens à préciser le peu que je sais du volumineux manuscrit inédit d'où est tirée la brève citation que j'analyse. Il s'agit du Journal de 2397 pages qui s'étend sur une dizaine d'années, entre 1920 et 1929. L'auteur en a parlé tantôt comme d'un pur exercice de style, tantôt comme du "livre de ma vie". Un autre passage de ce Journal réitère l'importance de la discipline qu'il représente pour le jeune écrivain:

J'ai soigné les phrases et fait un choix de mes expressions dans le dessein secret de leur plaire et de m'attirer l'encens de leurs vaines louanges. Aussi avais-je alors la précaution de traduire en une langue étrangère bien qu'harmonieuse les ressauts par trop personnels de ma sentimentalite. ${ }^{9}$

À part l'effort pathétique d'une séduction destinée à des lecteurs inconnus (faute de disposer d'un contexte suffisant), je retiens l'idée de relever la qualité de l'expression, idée exprimée presque dans les mêmes termes que dans le passage étudié tout à l'heure:

9 Cité par Jean Panneton, * Ringuet le moraliste ", dans Ringuet en mémoire 50 ans après Trente arpents, Québec, Éditions du Septentrion, 1989, p. 31. 
"la précaution de traduire en une langue étrangère. "N'est-il pas saisissant de retrouver ce souci de combler un écart présumé entre une expérience qui cherche à se dire et la fameuse langue étrangère qui pourrait la rendre lisible? Cette obsession circonscrit très exactement le lieu de la littérature dans la vocation du futur romancier. Le rapprochement avec le passage précédent ajoute à la solidité de l'hypothèse: le détestable patois qui exige une traduction, ce n'est pas seulement l'idiosyncrasie des paysans; c'est plutôt le fond excessif d'une sensibilité trop personnelle, inavouable sans le recours à la littérature, dont deux mots disent magnifiquement le mensonge paradoxal, celui d'^une langue étrangère bien qu'harmonieuse. . Écrire, c'est clairement échanger le poids d'une secrète vérité contre l'harmonie qui la sauve et l'allège, mais au prix de la livrer à l'étrangeté d'une autre «langue.. On voit bien que c'est la littérature qui tient cette fois la place de langue étrangère, mais on aperçoit surout en quel sens elle constitue une "langue . Peut-on mieux définir la cruelle nécessité qui appelle le sujet à l'écriture? Il y a d'ailleurs un autre texte, fort significatif, à rappeler à ce sujet. Peu après la publication de Trente arpents, Ringuet formule, dans un article destiné au public français, cette revendication d'autonomie de la littérature canadienne:

Nous nous estimerions heureux si, mettant en commun avec nos frères de France ce que nous pouvons avoir de personnel, nous parvenions à faire reconnaître, sans autre plébiscite, la littérature canadienne-française comme une province intellectuelle de la littérature française. ${ }^{10}$

Du Canada français à la France, l'espace littéraire ne peut-être que celui de la province à la capitale. L'écrivain canadien-français accepte, ou plutôt il réclame son rôle de périphérique. En vertu d'une logique sans doute analogue, le patient paysan doit accepter de voir traduire son patois sous la plume du médecin romancier.

C'est beaucoup prêter, j'en conviens, à de trop courts extraits dont j'ignore le contexte, sinon qu'ils appartiennent aux écrits intimes que l'auteur ne destinait pas à la publication. Ce qui m'intéresse, c'est que la position du diariste, pour autant qu'on puisse en juger sur un aussi mince échantillonnage, est loin d'être

10 Cité par Francis Parmentier, - La réception critique de Trente arpents dans la presse québécoise des années 1938-1939*, dans Ringuet en mémoire 50 ans apnès Trente arpents, op. cit., p. 81. 
44

sans rapports avec ce que l'on sait du romancier qu'il se préparait à devenir. Je n'essaie pas de situer Ringuet dans la querelle du régionalisme, mais de savoir si les idées de l'écrivain sur la langue ont vraiment joué un rôle dans son esthétique romanesque. Selon certains travaux qui permettent de mieux connaître la stratégie narrative de Trente arpents, il me semble que oui. Je pense, par exemple, à l'étude récente que Javier Garcia Mendes a consacrée à ce roman dans un chapitre de son essai intitulé la dimension bylique du roman. Le critique y écrit ceci:

Dans Trente arpents, l'omniloquence académicienne d'un narrateur, maitre absolu de la parole, met toutes les autres voix au rancart $[. .$.$] La traduction des discours des personnages$ montre le discours paysan davantage comme langue que comme langage et, plus précisément, comme une langue étrangère à celle du texte et, partant, à celle du lecteur. On comprend alors que ce dernier soit censé devoir être initié à cette langue ainsi qu'aux réalités et usages de la campagne par un narrateur qui devient tout à tour linguiste, anthropologue, sociologue. ${ }^{11}$

Beaucoụp de critiques ont déjà relevé la trace documentaire de Trente appents et la richesse des informations de toute sorte dont l'écrivain a surchargé son canevas fictif. Dans l'analyse de Javier Garcia Mendez, ces interventions du narrateur ne servent pas la qualité du roman, mais révèlent au contraire son échec esthétique. L'étendue du commentaire qui double les dialogues, étouffe la parole des personnages, interprète leurs silences et prête voix à leur mutisme, sous prétexte de les expliquer, cette prolifération paradiégétique témoigne d'une impuissance à intégrer la pluralité des discours dans le récit romanesque. Les protagonistes parlent, certes, et le romancier les fait s'exprimer "en patois "dans les dialogues, mais il les fait taire plus souvent encore, soit littéralement (leur incapacité de trouver les mots est presque un leitmotiv), soit en prenant la parole à leur place pour élucider leurs dires et éclairer leurs non-dits. Les effets de cette exégèse interne vont du paternalisme protecteur à la plus cinglante ironie. Bref, poursuit Javier Garcia Mendez, "la langue campagnarde québécoise ne s'est pas faite texte, elle n'est restée que chose à être observée, commentée à partir d'une autre langue qui est celle du roman "12.

11 Javier Garcia Mendes, La dimension bylique du roman, Montréal, Editions du Préambule, 1990, p. 115. 
Javier Garcia Mendez parle aussi de "la tendresse honteuse de Ringuet pour la parole de ses paysans"; le critique y entrevoit "peut-être une indication de la force avec laquelle, au Québec, ces "chaires, tribunes et journaux" dépositaires de l'éloquence nationale, dont Trente appents parle à la page 274, étouffent les voix populaires " ${ }^{13}$. Il reste encore beaucoup de choses à connaître sur l'énonciation des romanciers québécois d'avant 1960 et l'article de Garcia Mendez y contribue partiellement. À la suite du Romancier fictif ${ }^{14}$ d'André Belleau, qui étudiait la question du côté de l'écrivain personnage, Garcia Mendez l'examine du côté du narrataire ou du lecteur représenté dans la société textuelle. Il démontre brillamment, en démystifiant le personnage d'Albert Chabrol, que Ringuet a écrit son roman pour un public français, non pas parce que le livre est publié chez un éditeur parisien, mais parce que l'écriture romanesque déclare à qui elle s'adresse presque à chaque page. Ce choix du romancier entraîne des conséquences stylistiques, sémiotiques et diégétiques qui aboutissent à l'exclusion de la parole des personnage au moyen du discours omniscient d'un narrateur qui agit comme le tuteur des voix mises en dialogue. Garcia Mendez établit ce point sur des bases théoriques concluantes et par une lecture attentive du texte de Trente anpents. Ce n'est donc pas la peine d'y revenir. Je voudrais, pour conclure, ajouter quelques remarques complémentaires, en m'arrêtant à des interventions du narrateur qu'il n'a pas relevées.

L'une des plus remarquables et des plus longues sorties du narrateur de Trente arpents se trouve à peu près au milieu du récit, au début de la troisième partie ("L'automne"), lorsque l'unité familiale et la prospérité matérielle des Moisan commencent à subir les premières secousses d'un vent contraire qui va bientôt tout emporter. L'éclatement de la guerre et la profonde division qu'elle entraîne au sein de la dualité canadienne (la crise de la "conscription") forment l'arrière-plan des malheurs qui vont s'abattre sur la terre et la tribu du pauvre Euchariste. La fin du premier chapitre de "L'automne " consacre cinq pages à l'évocation des affrontements de Montréal enflammé par l'éloquence d'Henri Bourassa: deux pages de dialogues sont suivies par deux pages où le narrateur se transforme en éditorialiste pour désavouer carrément le sentiment exprimé par ses personnages. Les

14 Montréal, Presses de l'Université du Québec, 1980. 
46

acclamations populistes sont évidemment assumées par ceux-ci, qui n'ont pas l'air de ménager leur appui au célèbre tribun:

- Bourassa! dit Euthérius, c'est pas un manche d'alène! [...]

Et d'une voix lente dans le silence épais:

- I' paraît qu'i's vont les arrêter, lui pi Lavergne!

Stupeur! Il n'y eut qu'Ephrem que l'on entendit murmurer entre ses dents serrees:

- Ah ben! les crisses! 15

Le ton monte dans «la boutique de Deus* et Ephrem se dit prêt, s'il le faut, à résister par les armes à l'éventualité de la conscription. Mais lorsque le narrateur reprend le fil de son récit, le lecteur sait tout de suite à quoi s'en tenir sur ces gros mots: "Mais au fond toutes ces rodomontades n'avaient rien de bien dangereux. "16 L'antagonisme de la narration et du dialogue ne saurait être plus tranché. Joli mot que ces "rodomontades", et qui souligne que la langue du roman non seulement n'est pas celle qu'on vient d'entendre dans la bouche des paysans, mais surtout qu'elle dit tout autre chose. On ne saurait mieux saisir l'écart entre la langue qui parle et la langue qui écrit, c'est-à-dire entre le patois et la littérature. Cette différence est structurale dans Trente arpents. C'est elle qui fonde tout le système du récit. C'est elle qui en ruine aussi le réalisme, pourtant généralement acclamé.

Le problème n'est pas tant dans la divergence appuyée (encore que...) du narrateur et des personnages, que dans l'articulation même de ce rapport par le romancier. Car entre les deux perspectives opposées, le lecteur n'est pas libre de faire son propre choix: le narrateur se charge de lui indiquer clairement où se trouve la vérité. Non contente de s'exprimer bien haut dans la syntaxe narrative, cette vérité se présente encore comme subsumant le point de vue des protagonistes:

Quand la parole de Bourassa se fut soumise et que l'épiscopat fidèle à sa politique centenaire eut requis l'obéissance, il ne se trouva plus de voix qui fît connaître et expliquât à la fois le refus de tout un petit peuple brisé. Personne pour dire combien pourtant plus simple tout cela était.

Rivés au sol laurentien, le seul qu'ils connussent... ${ }^{17}$

15 Ringuet, Trente arpents, Paris, Editions J'ai lu, 1980, p. 176.

16 Ibid., p. 177.

17 Ibid., p. 177-178. 
Cette absence de voix pour faire connaître et expliquer "le refus de tout un petit peuple brisén, c'est donc le romancier qui va y suppléer, et tout invite à croire qu'il le fait au nom de ceux qui n'ont pas su fournir eux-mêmes l'explication. Le passage amène un long discours du narrateur qui établit l'indifférence à la guerre des "gens paisibles du Québec*, incapables de concevoir "d'autres soucis que [le] ciel immédiat au-dessus d'eux et, aussi, [le] prix de l'avoine et du foin "18. Cette énorme intervention dépasse de beaucoup la rectification des "rodomontades" du dialogue. Elle s'étend en réalité à toute la population québécoise englobée par la verbosité inoffensive de tous les Ephrem Moisan de la province. En d'autres mots, le narrateur sort carrément de son rôle de régisseur diégétique pour énoncer librement les opinions de l'auteur sur la réaction de ses compatriotes confondus dans le bloc compact des palabreurs de Saint-Jacques: "Combien pourtant plus simple que cela... "La leçon est assez claire, en effet! L'explication fait entendre un son tout différent de l'indignation d'Ephrem, qui proclamait, quelques pages plus haut: •Qu'i's viennent, maudit! Qu'ils viennent [...] Pi que j'aie pas seulement la chance d'en rencontrer un enfant de chienne, dans un coin, de ces tabernacles d'Anglais-là ${ }^{19}$ Celui qui jappe ne mord pas...

Il n'est pas question de reprocher à Ringuet d'avoir voulu mettre une sourdine à l'ardeur virulente d'Ephrem, dont je ne prétendrai pas que la voix est plus vraie que celle du narrateur qui le muselle. Je ne conteste pas la part de bravade inconséquente et purement verbale qu'il y a dans la colère d'Ephrem (qui s'exilera bientôt à White Falls, où il s'assimilera joyeusement à "ces tabernacles d'Anglais-là ", lui et toute sa famille). La question n'est pas là. Ce que je me demande, c'est ce que le docteur Panneton voulait dire en notant dans son Journal sa répugnance à prendre "l'accent, les formes verbales et les constructions des clients, surtout paysans ", alors qu'il associait ce pénible exercice à une traduction. Ce que j'en conclus, c'est que Trente arpents résulte de la démarche inverse et que Ringuet s'y est essentiellement appliqué à traduire ses paysans en roman, c'est-à-dire, selon lui, en français, occasion rêvée de dédommager le docteur Panneton du malaise qu'il avait éprouvé à se traduire lui-même

18. Ibid., p. 180.

19 Ibid., p. 176-177. 
48

en canayen pour mieux servir sa clientèle d'habitants. Tout vient de cette méprise ou de cette difficulté déjà vieilles: comment faire passer l'oralité locale dans l'expression littéraire, donc universelle? Ce dilemme, qui est au coeur de la littérature canadiennefrançaise depuis toujours, Ringuet lui donne une solution qui se veut l'antithèse de la solution régionaliste, mais il aboutit ironiquement à la même aliénation par un nouveau chemin: traduite, la parlure paysanne est remplacée par le savoir et la langue du romancier, qui substitue sa culture à l'indigence ignare et pittoresque de la parole indigène. C'est ainsi qu'une sociologie élémentaire résulte des contradictions dont la littérature se nourrit au contraire: le docteur, qui savait encore parler patois, aura été effacé par la traduction de Ringuet et le médecin de campagne qui inspirait son réalisme, aura eu raison de son roman. 